\title{
Temporal symmetric pulses and symmetry breaking in generalized saturable Kerr-like media
}

\author{
Jerzy Jasiński ${ }^{*}$ \\ Faculty of Physics, Warsaw University of Technology, Koszykowa 75, 00-662 Warszawa,
}

Received September 11, 2009; accepted September 30, 2009; published September 30, 2009

\begin{abstract}
In the paper the propagation of temporal pulses through generalized saturable nonlinear Kerr-like media is described analytically. The influence of symmetry conditions on relations between phase and amplitude is analyzed. These relations introduced into a canonical description of propagation enable a solution of the Euler-Lagrange equations. The accuracy of a canonical description is discussed.
\end{abstract}

In saturable Kerr-like media the envelope $U(z, t)$ of a pulse propagating along $z$-direction satisfies the generalized nonlinear Schrödinger equation (GNSE or NSE with higher-order effects) [1-3]:

$$
U_{z}+\frac{i k_{2} U_{t t}}{2}-i \varepsilon U+\gamma\left(2(\varepsilon U)_{t}+(\kappa-1) U \varepsilon_{t}\right)=0 .
$$

In Eq. (1) $k_{2}$ designates group velocity dispersion, $\varepsilon$ nonlinear permittivity, $\gamma$ - nonlinear dispersion coefficient and $\kappa-1$ (for real $\kappa$ ) - relative coefficient of retardation of nonlinear response due to Raman effects. The indexes after functions denote differentiation over the indicated coordinates. The initial three terms are symmetric with respect to time $t$ while the other two - antisymmetric. Nevertheless lack of symmetry Eq. (1) can possess solutions with symmetric amplitude $r(z, t)=|U(z, t)|=$ $r(z,-t)$ on condition that the phase profile is neither symmetric nor antisymmetric. Expressing $U$ by means of amplitude and phase $U(\mathrm{z}, t)=r(z, t) \exp (i \varphi(z, t))$ and writing the phase in the form of sum of antisymmetric and symmetric part $\varphi(\mathrm{z}, t)=\varphi^{(a)}(\mathrm{z}, t)+\varphi^{(s)}(\mathrm{z}, t)$ we obtain from (1):

$$
\begin{gathered}
r_{z}^{2}-k_{2}\left(r^{2} \varphi_{t}^{(s)}\right)_{t}=0, \\
k_{2}\left(r^{2} \varphi_{t}^{(a)}\right)_{t}-2 \gamma((\kappa+1) \varepsilon-\kappa F)_{t}=0 .
\end{gathered}
$$

where:

$$
F(I)=\int_{0}^{I} \varepsilon(I) d I
$$

(two other equations resulting from (1) have no simple interpretation). If $r^{2} \varphi_{t}$ vanishes sufficiently fast for $t \rightarrow \forall \infty$ (for instance when $r$ describes bright soliton) the first equation (2) gives conservation of total intensity:

$$
P(z)=\int_{-\infty}^{\infty} r^{2}(z, t) d t=\text { const }=P,
$$

\footnotetext{
${ }^{*}$ E-mail: jasinski@if.pw.edu.pl
}

while the second one expresses the antisymmetric part of the phase as a function of amplitude profile:

$$
\varphi^{(a)}(z, t)=\frac{2 \gamma}{k_{2}} \int_{0}^{t}\left((\kappa+1) \varepsilon\left(r^{2}(z, t)\right)-\frac{\kappa F\left(r^{2}(z, t)\right)}{r^{2}(z, t)}\right) d t
$$

Equation (1) has no analytic non-stationary solution. Nevertheless, applying the Lagrange method [4-6] we are able to describe the propagation of non-stationary pulses approximately. The real and imaginary part of GNSE (1) can be written in this method in the form:

$$
\frac{\partial \mathcal{L}}{\partial \xi}-\frac{\partial}{\partial z} \frac{\partial \mathcal{L}}{\partial \xi_{z}}-\frac{\partial}{\partial t} \frac{\partial \mathcal{L}}{\partial \xi_{t}}=\frac{\partial \mathcal{R}}{\partial \xi_{z}},
$$

( $\xi$ stands for $U$ and $U^{*}$ or $r$ and $\varphi$ ) and contains known from mechanics Rayleigh density function $\mathcal{R}$ [7] together with Lagrange density $\mathcal{L}$. Choosing:

$$
\begin{gathered}
\mathcal{L}=r^{2} \varphi_{z}-\frac{k_{2}\left(r_{t}^{2}+r^{2} \varphi_{t}^{2}\right)}{2}-F+\gamma\left((\kappa+1) r^{2} \varepsilon-\kappa F\right) \varphi_{t}, \\
\mathcal{R}=2 \gamma\left((\kappa+1) r^{3}\left(r_{t} \varphi_{z}+r_{z} \varphi_{t}\right) \varepsilon^{\prime}+r\left(r_{t} \varphi_{z}-r_{z} \varphi_{t}\right) \varepsilon\right), \\
\mathcal{H}=\frac{k_{2}\left(r_{t}^{2}+r^{2} \varphi_{t}^{2}\right)}{2}+F-\gamma\left((\kappa+1) r^{2} \varepsilon-\kappa F\right) \varphi_{t}
\end{gathered}
$$

( $\varepsilon^{\prime}$ means $d \varepsilon(I) / d I$ and $\mathcal{H}$ is Hamilton density) we can check the equivalence of Eqs. (6) and GNSE (1).

Integrating densities (7) over the plane $z=$ const we obtain lagrangian, Rayleigh function and hamiltonian:

$$
\begin{gathered}
L(z)=\int_{-\infty}^{\infty}\left(r^{2} \varphi_{z}^{(s)}-\frac{k_{2}}{2} r_{t}^{2}-\frac{k_{2} r^{2}}{2}\left(\varphi^{(a)}\right)^{2}-F\right) d t, \\
R(z)=\left(\frac{2 \gamma^{2}}{k_{2}} \int_{-\infty}^{\infty} G d t\right)_{z}, \\
H(z)=\int_{-\infty}^{\infty}\left(\frac{k_{2}}{2} r_{t}^{2}+\frac{k_{2} r^{2}}{2}\left(\varphi^{(a)}\right)^{2}+F\right) d t .
\end{gathered}
$$

Function $G=G\left(r^{2}(z, t)\right)$ appearing in integrand of $R(z)$ is:

$$
G(I)=\left(\kappa^{2}-1\right) \int_{0}^{I} \varepsilon^{2}(I) d I-\frac{\kappa^{2}}{I}\left(\int_{0}^{I} \varepsilon(I) d I\right)^{2} .
$$

Let us differentiate the definition of Hamilton density over $z$, next apply the Euler-Lagrange equations (6), 
express the antisymmetric part of the phase $\varphi^{(a)}$ using (5) and then integrate over plane $z=$ const. Doing so we derive the law of conservation $d H / d z+R=0$, so:

$$
H(z)+\frac{2 \gamma^{2}}{k_{2}} \int_{-\infty}^{\infty} G\left(r^{2}(z, t)\right) d t=\mathcal{E}=\text { const. }
$$

Therefore, in generalized saturable medium the conserved quantity is not pure hamiltonian $H$, but its sum with a term determined by Rayleigh function $R$.

Assume parabolic profile of phase function $\varphi^{(s)}(z, t)$ and amplitude $r(z, t)$ as the product of height function $b(z)$ and shape function $f$ (satisfying condition $f(0)=1$ ). Let $f$ depend on $z$ only by means of pulse width $w(z)$ :

$$
\begin{gathered}
\varphi^{(s)}(z, t)=\beta(z)+t^{2} \theta(z), \\
r(z, t)=b(z) f(t / w(z)) .
\end{gathered}
$$

The above assumptions give the Lagrange, Rayleigh and Hamilton functions in the form:

$$
\begin{gathered}
\frac{L}{b^{2} w}=C_{1} \beta^{\prime}+C_{c} w^{2}\left(\theta^{\prime}-2 k_{2} \theta^{2}\right)-\frac{C_{k} k_{2}}{2 w^{2}}-\int_{-\infty}^{\infty} \frac{F\left(b^{2} f^{2}(\tau)\right)}{b^{2}} d \tau, \\
R=\left(\frac{2 \gamma^{2}}{k_{2}} w \int_{-\infty}^{\infty} G\left(b^{2} f^{2}(\tau)\right) d \tau\right)_{z}, \\
\frac{H}{b^{2} w}=2 C_{c} k_{2} w^{2} \theta^{2}+\frac{C_{k} k_{2}}{2 w^{2}}+\int_{-\infty}^{\infty} \frac{F\left(b^{2} f^{2}(\tau)\right)}{b^{2}} d \tau,
\end{gathered}
$$

where coefficients $C_{c}, C_{k}$ and $C_{1}, \ldots, C_{3}$ are constant numbers denoting shape integrals:

$$
\begin{gathered}
C_{c}=\int_{-\infty}^{\infty} \tau^{2} f^{2}(\tau) d \tau, \quad C_{k}=\int_{-\infty}^{\infty} f^{\prime 2}(\tau) d \tau, \\
C_{n}=\int_{-\infty}^{\infty} f^{2 n}(\tau) d \tau: \quad n=1, \ldots, 3 .
\end{gathered}
$$

Any generalized coordinate $\xi$ satisfies the EulerLagrange equation $\partial L / \partial \xi-\partial / \partial z\left(\partial L / \partial \xi_{z}\right)-\partial / \partial t\left(\partial L / \partial \xi_{t}\right)=\partial R / \partial z$. For $\xi=\beta$ and $\xi=\theta$ the resulting equations are very simple:

$$
\begin{aligned}
& C_{1} b^{2} w=P, \\
& \theta=-\frac{w^{\prime}}{2 k_{2} w} .
\end{aligned}
$$

Comparing the first line of (14) and (4) we identify $P$ as total intensity. Two remaining equations obtained for $\xi=b$ and $\xi=w$ give two more relations - one expressing $\beta^{\prime}$ and the other being a second-order differential equation determining $w(z)$. This last equation integrating once gives the same relation as the law of conservation (10):

$$
\frac{C_{c} P w^{\prime 2}}{2 C_{1} k_{2}}+V(w)=\mathcal{E}
$$

with:

$$
V=\frac{C_{K} k_{2} P}{2 C_{1} w^{2}}+w \int_{-\infty}^{\infty} F\left(\frac{P f^{2}}{C_{1} w}\right) d \tau+\frac{2 \gamma^{2} w}{k_{2}} \int_{-\infty}^{\infty} G\left(\frac{P f^{2}}{C_{1} w}\right) d \tau
$$

Function $V=V(w(z))$ can be treated as potential and $\mathcal{E}$ as canonical energy.

The energy integral (15) can be solved giving a formula expressing inverse function of evolving pulse width:

$$
Z=\int_{w_{\min }}^{w(z)} \frac{d w}{\sqrt{\frac{2 C_{1} k_{2}}{C_{c} P}(\mathcal{E}-V(w))}}
$$

For cubic-quintic nonlinearity $\varepsilon(I)=\varepsilon_{2}\left(I-I^{2} / I_{\text {sat }}\right)$ we have:

$$
\begin{gathered}
F(I)=\varepsilon_{2}\left(\frac{I^{2}}{2}-\frac{I^{3}}{3 I_{\text {sat }}}\right), \\
G(I) \cong \varepsilon_{2}^{2} \frac{\left(\kappa^{2}-4\right) I^{3}}{12} .
\end{gathered}
$$

In $G(I)$ terms of order $I^{4} / I_{\text {sat }}$ and $I^{5} / I^{2}$ sat were neglected, what means that the obtained solution will be valid only for small $\gamma$ or small $I / I_{\text {sat }}$. But due to the above approximation potential (16) is given by a simple formula:

$$
V=\frac{C_{2} \varepsilon_{2} P^{2}}{2 C_{1}^{2} w}+\frac{C_{k} k_{2} P}{2 C_{1} w^{2}}+\frac{C_{3} \varepsilon_{2}^{2} P^{3}}{3 C_{1}^{3} k_{2} w^{2}}\left(\frac{\gamma^{2}\left(\kappa^{2}-4\right)}{3}-\frac{2 k_{2}}{3 \varepsilon_{2} I_{\text {sat }}}\right)
$$

and integration (16) is possible to perform analytically.

Two initial terms in (19) determine the potential in Kerr medium $V_{\text {Kerr }}$, the remaining two describe the influence of saturation and higher-order effects.

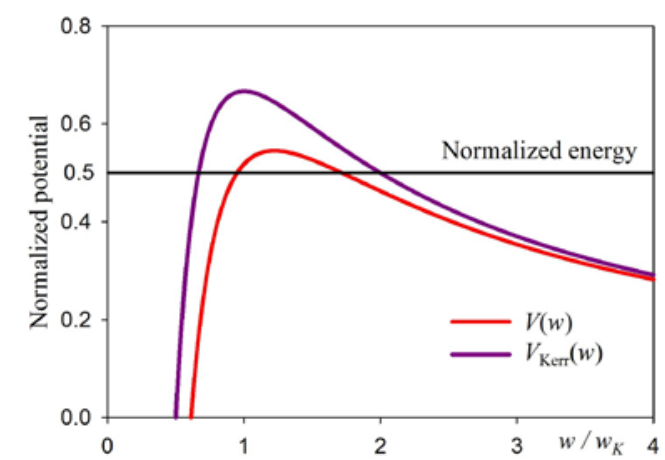

Fig. 1. Potential in Kerr and generalized medium.

In Fig. 1 we show the plots of potentials $V(w)$ and $V_{\text {Kerr }}(w)$ corresponding to the shape function given by:

$$
f(\tau)=\sqrt{\frac{1+\sigma}{\cosh ^{2} \tau+\sigma}}
$$

describing the amplitude of bright solitons in cubicquintic medium. A pulse propagating in such a potential oscillates - its width periodically changes between values lying at the intersection of potential and energy level E. The maximum of potential curves corresponds to 
stationary solutions - solitons. Their width and height are:

$$
\begin{gathered}
w_{s}=-\frac{2 C_{1}}{C_{2}}\left(\frac{C_{k} k_{2}}{\varepsilon_{2} P}+\frac{C_{3} \varepsilon_{2} P}{C_{1}^{2} k_{2}}\left(\frac{\gamma^{2}\left(\kappa^{2}-4\right)}{3}-\frac{2 k_{2}}{3 \varepsilon_{2} I_{s a t}}\right)\right), \\
b_{s}^{2}=\frac{P}{C_{1} w_{s}} .
\end{gathered}
$$

Integration (17) for potential (19) gives:

$$
\begin{aligned}
\frac{2 \pi z}{\Lambda}= & \arccos \left(\frac{w_{s}-\left(1-\Delta^{2}\right) w}{w_{s} \Delta}\right)- \\
& \sqrt{1-\Delta^{2}} \sqrt{\left(1-\frac{(1-\Delta) w}{w_{s}}\right)\left(\frac{(1+\Delta) w}{w_{s}}-1\right)}
\end{aligned}
$$

where two coefficients $\Delta$ and $\Lambda$ denote:

$$
\begin{gathered}
\Delta=\sqrt{1+\frac{8 C_{1}^{3} C_{k} k_{2} \mathcal{E}}{C_{2}^{2} \varepsilon_{2}^{2} P^{3}}+\frac{8 C_{1} C_{3} \mathcal{E}}{C_{2}^{2} k_{2} P}\left(\frac{\gamma^{2}\left(\kappa^{2}-4\right)}{3}-\frac{2 k_{2}}{3 \varepsilon_{2} I_{\text {sat }}}\right)}, \\
\Lambda=\frac{\pi C_{2} \varepsilon_{2}}{4} \sqrt{-\frac{2 C_{c} P^{5}}{C_{1}^{5} k_{2} \mathcal{E}^{3}}} .
\end{gathered}
$$

The solution (22) determines the pulse of a width $w$ between $w_{\min }$ and $w_{\max }$ expressed by:

$$
w_{\min , \max }=\frac{w_{s}}{1 \pm \Delta} \text {. }
$$

Since $\Delta=\left(w_{\max }-w_{\min }\right) /\left(w_{\max }+w_{\min }\right)$ we can interpret it as relative amplitude of oscillations. Analogously, $\Lambda$ is the period of oscillation.

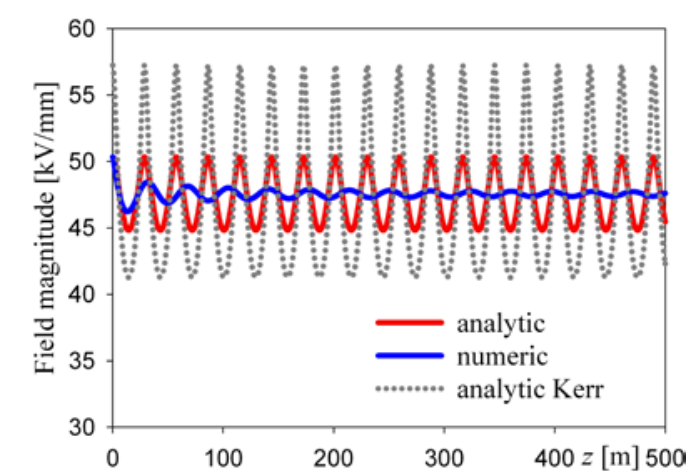

Fig. 2. Oscillations of pulse width $b(z)$ in generalized cubic-quintic medium. Comparison of analytic and numeric solution.

Comparing the obtained approximate analytic solution and the numeric one (Fig. 2) we observe that the field height calculated numerically agrees with analytic formula (20) only at very beginning. During propagation the amplitude of oscillations significantly diminishes and the period increases, although its changes are not so large. The disagreement is not caused by higher-order terms in GNSE, appearance of Rayleigh function in the EulerLagrange equations or applied approximation in potential
(19) - we shall observe a very similar behavior considering the propagation of Sech-pulse in pure Kerr medium. On the contrary, by choosing a more appropriate profile (20) instead of Sech (however for considered material parameters the widening coefficient $\sigma$ is small $\sigma=0.061$ ), the amplitude of oscillations diminished twice (as we can observe comparing dotted grey and solid red line in Fig. 2) and stationary values (21) correspond almost exactly to numeric ones. But the investigation of numeric phase profile leads to the conclusion that it is not parabolic, as assumed in the applied method (Fig. 3).

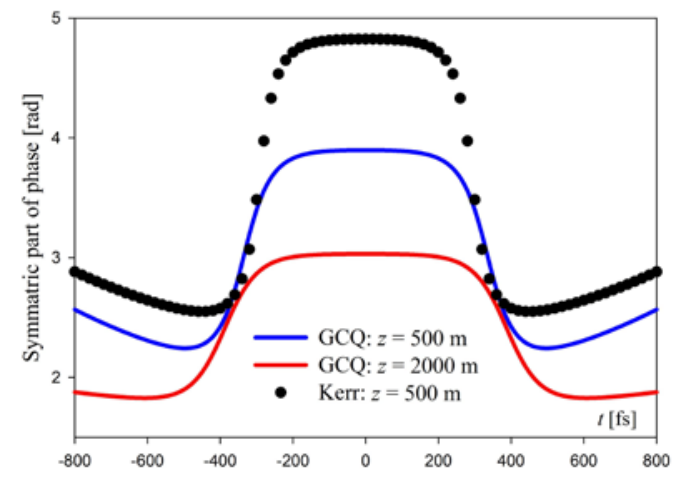

Fig. 3. Numeric phase profile in Kerr and generalized medium.

The second reason of disagreement is pulse spreading, nevertheless the total intensity $P$ is conserved. Both mentioned reasons mean, that an authentic field should be described by a much more complicated function than given by (19) and (10). Unfortunately, the complication of a trial function causes the Lagrange function (9) to be much more complex. Also by increasing the number of pulse parameters we increase the number of the EulerLagrange equations. As a result, the problem can be unsolvable analytically.

To summarize, the use of the Rayleigh function in the Euler-Lagrange equations and the application of adequate symmetry conditions causes better description of propagating pulses. But the neglect of certain effects important during pulse evolution results in not all pulse parameters being calculated exactly - some of them agree only roughly.

\section{References}

[1] K. Hizanidis, D. J. Frantzeskakis, C. Polymilis, J. Phys. A, 29, 7687, (1996)

[2] F.G. Bass, V.V. Konotop, S.A. Puzenko, Phys. Rev. A 46, 4185, (1992)

[3] J. Jasiński, Opt. Comm. 172, 325, (1999)

[4] D. Anderson, Phys. Rev. A, 27, 3135, (1983)

[5] C. Sulem, P.L. Sulem, The Nonlinear Schrödinger Equation: SelfFocusing and Wave Collapse, (New York, Springer-Verlag, 1999)

[6] G.P. Agrawal, Lightwave technology:telecommunications systems, pp. 137-142, Wiley Interscience, Hoboken, 2005

[7] H. Goldstein, C. Poole, J. Safko, Classical Mechanics, (Addison Wesley, San Francisco, 2000) 